\title{
Dispersion-Tuned Harmonically Mode-Locked Fiber-Optical Parametric Oscillator
}

\author{
Sigang Yang, Kim K. Y. Cheung, Yue Zhou, and Kenneth K. Y. Wong
}

\begin{abstract}
The stable operation of a high-repetition-rate dispersion-tuned harmonically mode-locked fiber-optical parametric oscillator (FOPO) is demonstrated for the first time to the best of our knowledge. The experimental results match well with the theory. By introducing large cavity dispersion, the round-trip delay can vary with wavelength. Thus the cavity can be synchronized with the modulation frequency of an external clock passively. The FOPO can be tuned simply by adjusting the modulation frequency. A stable 10-GHz pulse train is obtained over 6-nm tuning range for each of the signal and idler.
\end{abstract}

Index Terms-Nonlinear optics, optical fiber lasers, parametric amplifiers, parametric oscillators.

\section{INTRODUCTION}

$\mathbf{P}$ ARAMETRIC oscillators based on optical parametric amplification (OPA) in optical fibers have attracted significant interest recently because they provide tunable coherent radiation in a remarkable tuning range [1]-[3]. To obtain sufficient gain, the required cavity length for fiber-optical parametric oscillators (FOPOs) is typically tens, or even hundreds, of meters, in which case the frequency spacing between the longitudinal modes is extremely small, typically in the order of megahertz $(\mathrm{MHz})$ [4]. It is difficult to make an intracavity optical filter to select and track a single frequency; as a result, multiple longitudinal modes oscillate simultaneously. Usually the phases of these multiple longitudinal modes have random relationships [5]. To solve this problem, we have demonstrated an active mode-locking method to lock the phase of the multiple longitudinal modes [6]. Due to the temperature variation or environmental disturbance, the cavity readily drifts and loses synchronization easily. Therefore, the active stabilization of the cavity is necessary. One approach is to fix the repetition rate and allow the wavelength to vary in a cavity with a sufficient amount of dispersion [7]. As the round-trip time for a pulse inside the cavity depends on its wavelength in a dispersion cavity, introducing a large dispersion inside the cavity will enable the pulse to be synchronized with the external modulation frequency automatically. The reason is that the laser wavelength will adjust to center the pulse in the modulation transmission window [8].

Manuscript received November 04, 2009; revised January 26, 2010; accepted February 03, 2010. First published February 22, 2010; current version published March 24, 2010. This work was supported in part by grants from the research Grants Council of the Hong Kong Special Administrative Region, China (Project HKU7179/08E and HKU7183/09E).

The authors are with Photonic Systems Research Laboratory, Department of Electrical and Electronic Engineering, The University of Hong Kong, Hong Kong, SAR (e-mail: sgyang@eee.hku.hk; h0519406@eee.hku.hk; yzhou@eee. hku.hk; kywong@eee.hku.hk).

Color versions of one or more of the figures in this letter are available online at http://ieeexplore.ieee.org.

Digital Object Identifier 10.1109/LPT.2010.2043091

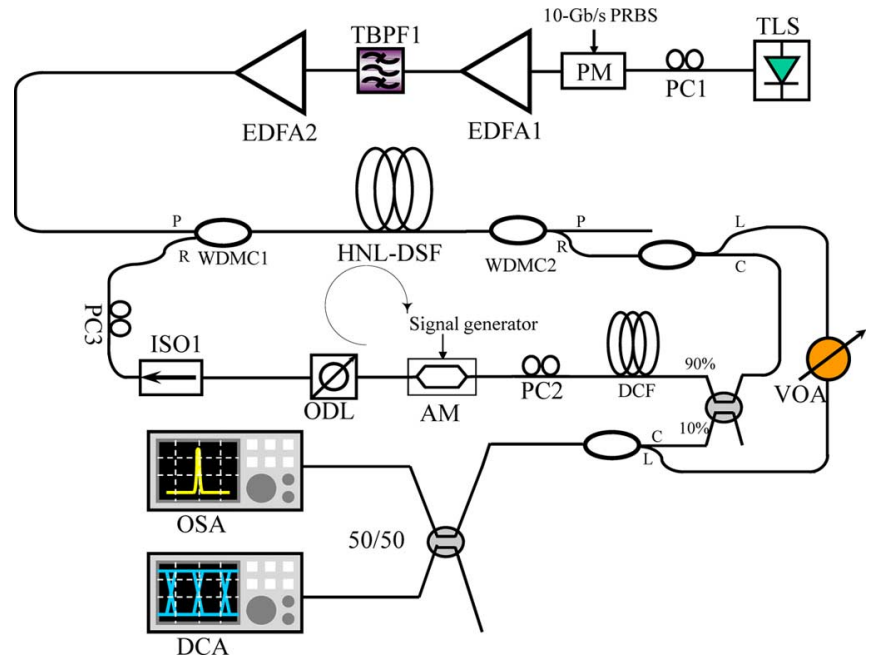

Fig. 1. Schematic diagram of the dispersion-tuned harmonically mode-locked FOPO.

This concept can be applied to the harmonically mode-locked FOPO. Here we report a stable operation of a $10-\mathrm{GHz}$ dispersion-tuned harmonically mode-locked FOPO, which matches well with the theoretical results, for the first time to the best of our knowledge. The FOPO can be tuned simply by adjusting the external modulation frequency and it can generate two pulse trains at the signal and the idler wavelengths. By utilizing an automatic scanning frequency from an external clock, it has a potential to be developed as a fast sweeping pulsed source.

\section{EXPERIMENTAL SETUP}

The schematic diagram of the dispersion-tuned harmonically mode-locked FOPO is shown in Fig. 1. The pump source was an external cavity tunable laser source (TLS). To suppress the stimulated Brillouin scattering (SBS), the light was first phase-modulated with a $10-\mathrm{Gb} / \mathrm{s}$ pseudorandom bit sequence (PRBS) signal via a phase modulator (PM). The polarization controller (PC), PC1, aligned the state of polarization (SOP) of the pump with the transmission axis of the PM. The SBS could be suppressed by up to $28 \mathrm{~dB}$. Then the pump was amplified by a two-stage configuration of erbium-doped fiber amplifiers (EDFAs). The maximum average pump power could reach as high as $33 \mathrm{dBm}$. The pump was afterward coupled into a $400-\mathrm{m}$ highly nonlinear dispersion-shifted fiber (HNL-DSF) via the P-port (1554.89-1563.89 $\mathrm{nm}$ ) of a bandpass wavelength-division multiplexing (WDM) coupler (WDMC1). The high power pump propagated through the HNL-DSF and was then coupled out of the cavity via the P-port of another bandpass WDM coupler (WDMC2), while the amplified signal and idler were propagated through the R-port (1500-1551 nm, 1567-1620 nm) 
of WDMC2. A $C / L$-band WDM coupler was inserted such that only the $C$-band signal was circulated inside the cavity while the idler was coupled out via the $L$ port. A 10/90 coupler was used to extract the output signal. The intracavity group-velocity dispersion (GVD) was introduced by a 200-m dispersion-compensation fiber (DCF) with a dispersion of $-18.56 \mathrm{ps} / \mathrm{nm}$ at $1550 \mathrm{~nm}$. A $\mathrm{LiNbO}_{3}$ Mach-Zehnder amplitude modulator (MZ-AM) driven by a signal generator was connected after the DCF. Due to the polarization dependence of the MZ-AM, a polarization controller (PC2) was used to adjust the SOP of the signal pulse. A tunable optical delay line (ODL), ranging from 0 to $350 \mathrm{ps}$, was used to adjust the cavity length. The isolator (ISO1) ensured unidirectional operation. PC3 was used to align the signal's SOP with that of the pump so as to maximize the signal gain. The output signal from the $10 \%$ port of the 10/90 coupler was then combined with the attenuated idler output via another $C / L$-band WDM coupler. After that the combined light was divided into two equal parts through a 50/50 coupler, which were then sent to an optical spectrum analyzer (OSA) and a digital communication analyzer (DCA), respectively.

\section{EXPERIMENTAL RESULTS AND DisCUSSION}

The HNL-DSF used in the experiment had a nonlinear coefficient of $14 \mathrm{~W}^{-1} \cdot \mathrm{km}^{-1}$ and its zero-dispersion wavelength was $1554 \mathrm{~nm}$. The OPA worked in its anomalous dispersion regime since the pump wavelength was $1556 \mathrm{~nm}$. We used amplified spontaneous emission (ASE) from EDFA as the seed source and inferred the OPA gain spectrum from the measurement of the output ASE spectrum after the fiber-optical parametric amplifier (FOPA) [9]. It demonstrated that for a continuous-wave (CW) pump at $1556 \mathrm{~nm}$, the maximum gain occurred at the signal wavelength of $1542 \mathrm{~nm}$ and the idler wavelength of $1570 \mathrm{~nm}$, respectively. However, the 3-dB gain bandwidth for both gain bands is $10 \mathrm{~nm}$ only.

The proposed FOPO had a cavity loss of $15.3 \mathrm{~dB}$. Once the OPA gain exceeded the cavity loss, oscillation would begin. Note that no intracavity filter was used to restrict the oscillation wavelength. Instead, a spool of DCF was inserted inside the cavity. The HNL-DSF was pumped by a $1.13-\mathrm{W}$ pump power and the modulator was driven by a $10.00008-\mathrm{GHz}$ sinusoid from the signal generator. When the radio-frequency (RF) signal was applied, mode locking was established automatically. Hence the FOPO began to oscillate. It could be monitored by a sharp enhancement in the output power. The power of the output signal was $5.9 \mathrm{dBm}$. The optical spectrum of the combined output signal and idler are shown in Fig. 2. It consists of a peak due to the residual pump, the oscillating signal at $1540 \mathrm{~nm}$, the generated idler at $1572 \mathrm{~nm}$, and spurious four-wave-mixing (FWM) peaks. As can be observed, only certain specific wavelengths in the cavity can oscillate even though there is no intracavity filter. Due to the large intracavity dispersion, different wavelengths experience different effective cavity length. In a cavity with a sufficient amount of dispersion, for a fixed external clock, only certain wavelengths can be synchronized with the external clock and oscillate. The intracavity dispersion introduced here acts as the mode selection element. Fig. 3(a) shows the waveform of the combined signal and idler. The full-width at half-maximum (FWHM) pulsewidth is $32 \mathrm{ps}$ and the jitter root mean square (rms) is 1.26 ps. Fig. 3(b) and (c) show the waveform of the

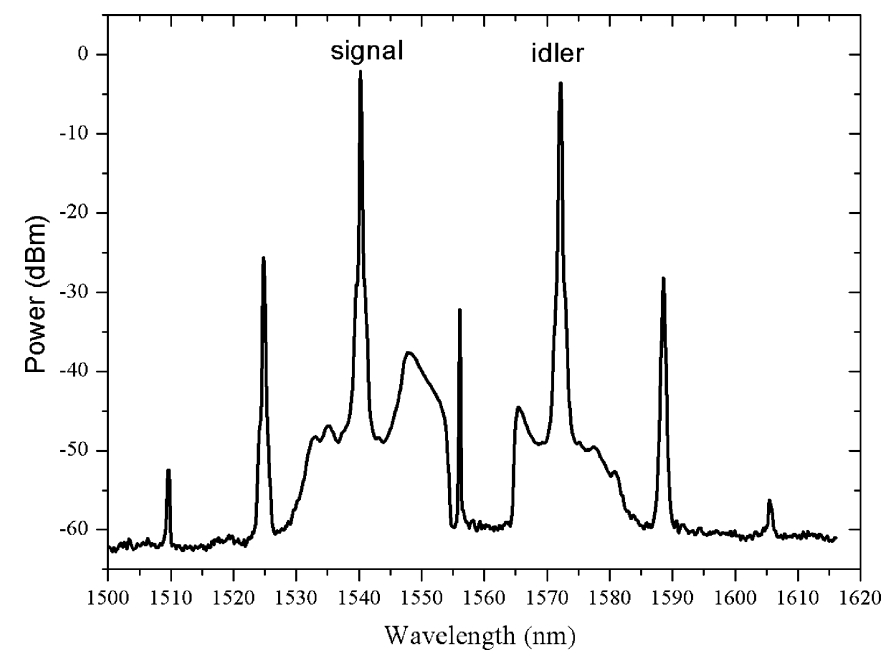

Fig. 2. Optical spectrum of the combined signal and idler.

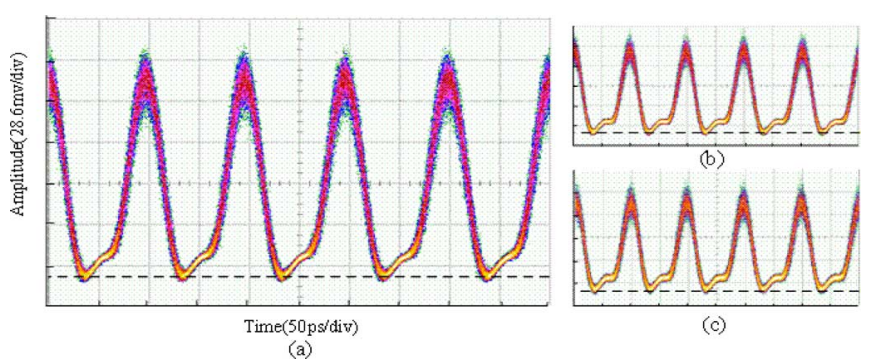

Fig. 3. Observed waveform from DCA. (a) The combined signal and idler; (b) the signal; (c) the idler.

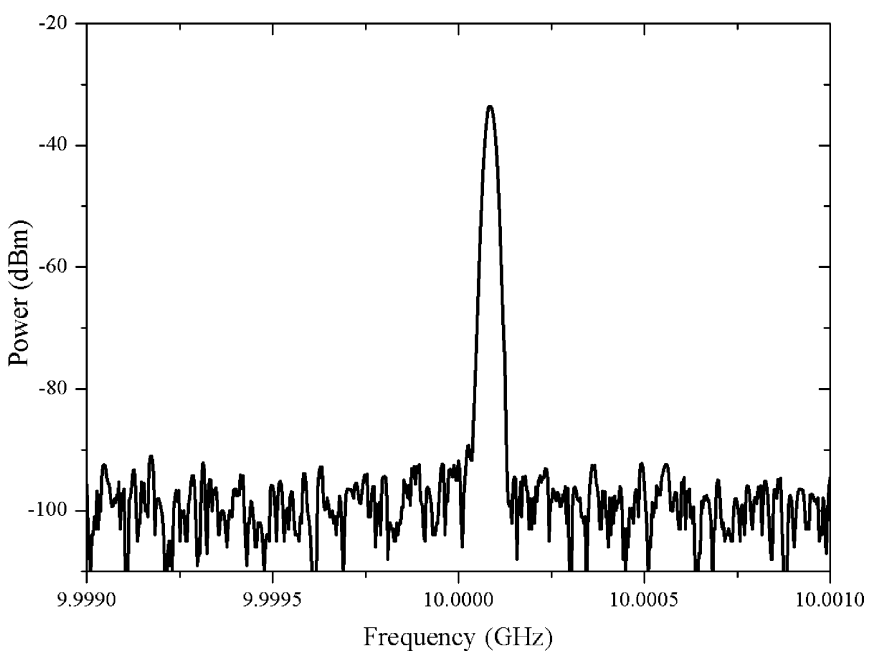

Fig. 4. RF spectrum at $10.00008 \mathrm{GHz}$ on a $2-\mathrm{MHz}$ span.

signal and idler, respectively. Hence, the proposed FOPO can provide synchronous pulse trains at two wavelengths.

Fig. 4 shows the RF spectrum of the output after detection with a high-speed photodiode (PD). Only the $10.00008-\mathrm{GHz}$ component can be observed at 2-MHz span. Pulse-to-pulse fluctuations could appear as noise components at harmonics of the fundamental cavity frequency $(330 \mathrm{kHz})$. Such fluctuations were suppressed to less than $-90 \mathrm{dBm}$.

The stability of the harmonically mode-locked FOPO was also investigated experimentally. The output pulse displayed in 


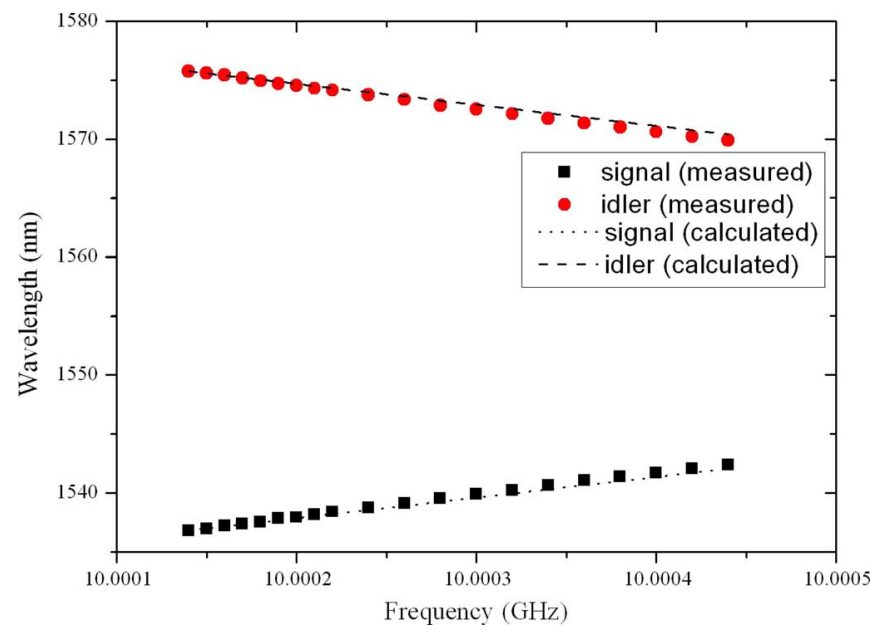

Fig. 5. Tunable characteristics of the dispersion-tuned harmonically mode-locked FOPO.

DCA was observed for $2 \mathrm{~h}$. There was no observable pulse shape variation, except that the pulse delayed by $30 \mathrm{ps}$ after $2 \mathrm{~h}$. The reason for the time delay of the pulse train was due to the drift of the cavity. It can be shown that the proposed FOPO can adjust its oscillating wavelength automatically so as to maintain synchronization. The new pulse train is time-delayed compared with the original pulse train due to the dispersion effect in the cavity.

For a dispersion-tuned harmonically mode-locked FOPO, smooth wavelength tuning can be achieved by changing either the modulating frequency or the cavity length without disturbing the stable pulsing state. We first fixed the cavity length and tuned the modulating frequency of the signal generator. During the tuning process, a stable pulse train had both its temporal and spectral widths preserved. Fig. 5 shows the tunable characteristics of the dispersion-tuned FOPO when the external modulating frequency was adjusted. When the modulating frequency was increased, the signal would shift to the longer wavelength linearly while the idler shifted in the opposite direction. When the modulating frequency was tuned from 10.00014 to $10.00044 \mathrm{GHz}$, the signal light had to adjust its own wavelength in order to synchronize itself with the external clock. Since the signal light is located at the normal dispersion regime, red shift will occur while blue shift will occur for the idler side. The measured tuning slope of $18.67 \mathrm{~nm} / \mathrm{MHz}$, related to the cavity dispersion, is consistent with following derivation. The tuning sensitivity can be calculated according to [10] as: $S=-n_{0} /\left(c D f_{m 0}\right)$, where $n_{0}$ is the equivalent refractive index of the core at a reference wavelength, $c$ is the velocity of light in free space, $D$ is the equivalent dispersion parameter, and $f_{m 0}$ is the modulation frequency. The calculated value is $17.5 \mathrm{~nm} / \mathrm{MHz}$, which matches very well with the experimental results, as shown in Fig. 5. The tuning range is $6 \mathrm{~nm}$, which is approximately limited by the gain bandwidth of FOPA and the competition from other oscillation modes. Compared with the traditional tuning mechanism, this scheme only requires adjustment in the external modulation frequency. If the signal generator can support a swept RF signal, the FOPO can provide fast sweeping light sources at two wavelengths. Therefore, by utilizing an automatic scanning frequency from an external clock, it can potentially be developed as a fast sweeping pulsed source.

\section{CONCLUSION}

We have demonstrated a dispersion-tuned harmonically mode-locked FOPO. By introducing large intracavity dispersion, the cavity can self-synchronize to an external clock automatically by the dispersion-induced self-tuning. Stable 10-GHz pulse trains at two wavelengths with a pulsewidth of 32 ps are generated. Moreover, it is capable of maintaining synchronization regardless of the drifts of cavity length. By tuning the external modulation frequency, smooth wavelength tuning is achieved over $6 \mathrm{~nm}$ with stable pulse shape, in both temporal and spectral domains. It can potentially be developed as a fast sweeping pulsed source by utilizing a fast scanning microwave signal.

\section{ACKNOWLEDGMENT}

The authors would like to acknowledge Sumitomo Electric Industries for providing the HNL-DSF.

\section{REFERENCES}

[1] J. E. Sharping, "Microstructure fiber based optical parametric oscillators," J. Lightw. Technol., vol. 26, no. 14, pp. 2184-2191, Jul. 15, 2008.

[2] G. K. L. Wong, S. G. Murdoch, R. Leonhardt, J. D. Harvey, and V. Marie, "High-conversion-efficiency widely-tunable all-fiber optical parametric oscillator," Opt. Express, vol. 15, no. 6, pp. 2947-2952, 2007.

[3] Y. Zhou, K. K. Y. Cheung, S. Yang, P. C. Chui, and K. K. Y. Wong, "Widely-tunable picosecond optical parametric oscillator using highlynonlinear fiber," Opt. Lett., vol. 34, no. 7, pp. 989-991, 2009.

[4] M. E. Marhic, Fiber Optical Parametric Amplifibers, Oscillators and Related Devices. Cambridge, U.K.: Cambridge Univ. Press, 2008

[5] O. Svelto, Principles of Lasers. New York: Plenum Press, 1998.

[6] S. Yang, Y. Zhou, J. Li, and K. K. Y. Wong, "Actively mode-locked fiber optical parametric oscillator," IEEE J. Sel. Topics Quantum Electron., vol. 15, no. 2, pp. 393-398, Mar./Apr. 2009.

[7] S. Li and K. T. Chan, "Electrical wavelength tunable and multiwavelength actively mode-locked fiber ring laser," Appl. Phys. Lett., vol. 72, pp. 1954-1956, 1998.

[8] K. Tamura and M. Nakazawa, "Dispersion-tuned harmonically modelocked fiber ring laser for self-synchronization to an external clock," Opt. Lett., vol. 21, pp. 1984-1986, 1996.

[9] M. E. Marhic, K. K. Y. Wong, and L. G. Kazovsky, "Wide-band tuning of the gain spectra of one-pump fiber optical parametric amplifiers," IEEE J. Sel. Topics Quantum Electron., vol. 10, no. 5, pp. 1133-1141, Sep./Oct. 2004.

[10] Y. Nakazaki and S. Yamashita, "Fast and wide tuning range wavelength-swept fiber laser based on dispersion tuning and its application to dynamic FBG sensing," Opt. Express, vol. 17, no. 10, pp. 8310-8318, 2009. 